\title{
Research Progress of TCM External Treatment of Knee Osteoarthritis
}

\author{
Fangzong Deng ${ }^{1}$, Xuefeng Pang ${ }^{2, *}$, Yun Li ${ }^{1}$ \\ ${ }^{2}$ Ruikang Hospital affiliated to Guangxi University of Chinese Medicine, Nanning 530001, China \\ *Corresponding author
}

\begin{abstract}
Knee osteoarthritis is one of the most common osteoarthropathies in clinical practice. It is often found in middle-aged and elderly people. Its clinical manifestations include knee stiffness and pain, lower limb dysfunction and activity limitation, claudication, and even inability to walk. This article reviews the application of external treatment of Traditional Chinese Medicine in knee osteoarthritis in recent years, summarizes the research progress of external treatment, to provide more reference for clinicians in the treatment of knee osteoarthritis.
\end{abstract}

Keywords: Knee osteoarthritis, Understanding of knee osteoarthritis in Traditional Chinese Medicine, External treatment of Traditional Chinese Medicine.

\section{Knee Osteoarthritis}

Knee osteoarthritis (KOA) is a degenerative degeneration of knee cartilage, followed by osteoporosis, proliferation, and joint inflammation. The symptoms are mainly manifested as local pain, swelling, impaired joint function, and even quadriceps atrophy[1]. Domestic studies suggest[2] that the probability of developing knee arthritis increases with age. The incidence rate in females is higher than that in males, especially after the age of 50, which may be caused by hormonal imbalance after menopause[3]. In recent years, Traditional Chinese Medicine has made great progress in the treatment of KOA, and many safe and effective prescriptions and treatment methods have emerged, mainly including internal administration and external treatment[4]. The comprehensive treatment of Traditional Chinese Medicine, such as internal and external application of Traditional Chinese Medicine, acupuncture, moxibustion, manipulation and massage, rehabilitation training, etc.[5] has obvious effects.

\section{Understanding of Knee Osteoarthritis in Traditional Chinese Medicine}

Knee osteoarthritis belongs to "bi syndrome" and "bi bone" in Traditional Chinese Medicine, and also has nicknames such as "calendar disease" and "crane knee wind". Huangdi Neijing, a classic medical book of Traditional Chinese Medicine, first put forward the concept of "bi syndrome", which is divided into bone bi, running bi, tendon bi, pain bi, bi, zhou $\mathrm{Bi}$, and calendar festival wind. For example, in $\mathrm{Su}$ Wen · Bi Syndrome, it says, "Wind, cold and wet three qi come together to form bi."

\section{The Doctor of Traditional Chinese Medicine External Treatment}

External treatment of Traditional Chinese Medicine is to stimulate specific acupoints, meridians, muscles, and bones of patients with the assistance of relevant equipment or manipulation, to achieve the effect of curing diseases and removing evil. At present, the external treatment of Traditional Chinese Medicine for KOA mainly includes acupuncture, moxibustion, acupotomy, cupping, manipulation, external application, and Traditional Chinese Medicine fumigation[6].

\subsection{Acupuncture}

\subsubsection{Ordinary acupuncture}

Tu Jianfeng et al.[7] observed the pain, symptoms (except pain), daily activities, sports and entertainment functions, and quality of life of patients in the acupuncture group and sham acupuncture group before and after treatment, and concluded that acupuncture treatment could improve joint pain and daily activities of patients with knee osteoarthritis.

\subsubsection{Acusector}

Electroacupuncture is a kind of therapy to treat diseases by stimulating acupoints with electric current based on acupuncture to get qi. Yin Yueshan et al.[8] added electroacupuncture to oral glucosamine sulfate therayand found that VAS score, WOMAC score, SF-36 score, and muscle strength around knee joint were improved compared with before treatment. Clinical studies at home and abroad have shown $[9,10,11]$ that electroacupuncture can effectively relieve pain, joint stiffness, and dysfunction caused by KOA, and has the advantages of quick onset, good long-term efficacy, safety, and economy. Domestic researchers[12] randomly divided 100 patients with knee osteoarthritis into the observation group and the control group. Patients in the control group were treated with electroacupuncture combined with knee functional exercise, and patients in the observation group were treated with local Chinese medicine external application of the knee based on the control group. It was found that the total effective rate in the observation group was $96.00 \%$, higher than that in the control group (82.00\%).

\subsubsection{Fire needle}

In Huangdi Beijing (Yellow Emperor's Inner Canon), the needle is called "burnt prick". The needle body is burned red and pointed at the acupoint to prick and prick, preventing and curing diseases through searing stimulation. Fu D Y [13] found through experiments that fire needles can increase 
serum MMP-3 and IL- $1 \alpha$ levels of patients, and can improve the symptoms of pain, stiffness, and joint mobility disorders in mild to moderate KOA patients. Xie Chuan et al.[14] divided patients with knee osteoarthritis into combination group and meloxicam tablet group. Patients in the meloxicam tablet group were treated with meloxicam tablet alone, while patients in the combined group were treated with hao Huo needle based on meloxicam tablet. The combined treatment with meloxicam could significantly reduce WOMAC and VAS scores. Some researchers[15] used the method of firing needle to treat yang-deficiency and cold coagulation knee osteoarthritis, and used the method of firing needle to quickly prick relevant acupoints around the affected side of the knee, and found that the effective rate was $93.33 \%$, and the VAS pain score and WOMAC score could be reduced.

\subsubsection{Moxibustion}

Moxibustion means to stimulate specific acupoints of the human body with the help of the heat of moxibustion fire, which has the effect of warming the meridian and dispersing cold, warming Yang, and tonifying deficiency. Chen Yu et al.[16] tested the levels of TNF- $\alpha$, IL-1 $\beta$, MDA, and SOD activity in serum of patients in the acupuncture group and the moxibustion group before and after treatment, and suggested that both moxibustion and acupuncture treatment could alleviate KOA symptoms, and the efficacy of moxibustion was better than that of acupuncture. Yang Liu et al.[17] proved that different moxibustion methods can relieve the knee joint symptoms of PATIENTS with KOA, but the mechanisms of action are different. For example, heat-sensitive moxibustion focuses on the meridian transmission and heat-sensitive effect on acupoints. Thunder fire moxibustion has strong medicinal force, strong penetration, large area, high thermal radiation, and wheat grain moxibustion has an obvious analgesic effect on the outside.

\subsubsection{Thunder fire moxibustion}

The principle of thunder fire moxibustion is to improve local blood transport and play an anti-inflammatory and swelling effect by burning moxa sticks containing special drugs under the effect of light and heat radiation. An experimental study showed that thunder fire moxibustion can effectively regulate the expression of MMP-3 and TIMP-1 and up-regulate the level of TGF- $\beta 1$, to delay the lesions of articular cartilage and promote the removal of inflammatory mediators, which is the potential mechanism of its therapeutic effect[18]. Relevant studies suggest that thunder fire moxibustion combined with celecoxib can significantly improve the symptoms and signs of KOA patients, proving that thunder fire moxibustion can enhance the recovery of joint function and relieve pain[19]. Zhao Min et al.[20] applied guide Peony Zhimu Decoction plus subtraction combined with thunder fire moxibustion in the treatment of knee osteoarthritis, with an effective rate of $93.42 \%$, which could improve the gait parameters and bone metabolism of PATIENTS with KOA and relieve the clinical symptoms of patients. Tian Aiping[21] of 94 cases of patients with knee osteoarthritis were randomly divided into treatment group and control group, the treatment group was treated in the control group given on based on living by thunder fire moxibustion combined parasitic soup treatment, treatment group, treatment can improve patients' pain symptoms, patients with knee joint function, improve the level of the serum levels of inflammatory cytokines, thus improve the patients quality of life. Cui Fan et al.[22] proposed that thunder fire moxibustion combined with sodium hyaluronate injection in the joint cavity can significantly improve knee function and reduce KOA recurrence in the treatment of knee osteoarthritis. Hu Jun et al.[23] proposed in the experimental study that thunder fire moxibustion treatment has a positive effect on improving the clinical symptoms of KOA. In addition, thunder fire moxibustion cream can make patients have a more lasting skin thermal sensation in the treatment of $\mathrm{KOA}$, to better improve the clinical symptoms of KOA.

\subsubsection{Thermal moxibustion}

Thermal effect was put forward by professor Xin Chen, etc in recent years of a kind of brand-new moxibustion methods, full name "acupoints heat-sensitive suspended moxibustion therapy", related acupoints to produce special induction heat, namely the phenomenon of "little irritation reaction[24] Wenxue Wang[25], thermal moxibustion therapy in patients with knee osteoarthritis, and through the visual analog scale to assess patients before and after treatment level of pain, It was found that the effective rate of heat-sensitive moxibustion group was 90\%. Fang Xianfeng et al.[26] applied heat-sensitive moxibustion combined with oral Chinese medicine for strengthening liver and kidney muscles and bones to treat 50 cases of knee osteoarthritis, and the results showed that VAS joint pain score, Lysholm score, and fastest walking time of 50 yards were significantly improved compared with before treatment, indicating that heat-sensitive moxibustion is effective.

\subsection{Cupping}

Cupping therapy has the functions of regulating channels and activating collaterals, promoting qi and blood circulation, dispelling wind, and dispersing cold. The mechanism of cupping may be related to the change of local energy metabolism and local neuro-immune regulation mechanism[27]. Qiu Jianqing etc.[28] by comparing the control group and treatment group in improving clinical symptoms integral, pain score, improving the joint movement function, regulating inflammatory cytokines IL - 1, the content of TNF alpha, etc., concluse: using acupuncture combined cupping therapy, in improving the clinical symptoms and joint motion function, accelerate the effusion absorption has a certain advantage.

\subsection{Manipulative Therapy}

Manipulation refers to the effect of relaxing tendons and collaterals, activating blood circulation and removing blood stasis, releasing adhesions and smoothing joints using point pressing, kneading, holding and stretching and other tendon and bone consolidation techniques, which can improve joint stiffness and muscle strength, relieve joint pain and improve joint function. Jiang Liming et al.[29] showed that the release of the subpatellar fat pad can effectively treat knee osteoarthritis, thus alleviating the clinical symptoms of patients and further improving their quality of life. Domestic studies have confirmed[30] that chiropractic therapy from the 
waist is a simple and effective treatment for knee osteoarthritis.

\subsection{Topical Therapy}

Quan Xiaoming et al.[31] compared the total treatment efficiency of the observation group and the control group and found that the external application of Shuangbai Powder had better efficacy than pure thunder fire moxibustion. Wang Zheng et al.[32] randomly divided 60 patients with early and middle stage knee osteoarthritis into observation group (external application of Xiaoyu Jiegu SAN) and control group (oral celecoxib capsule), 30 cases in each group, and found that external application of Xiaoyu Jiegu SAN had obvious efficacy in improving clinical symptoms and knee function.

\subsection{Needle-knife Therapy}

Autonomy means that the surgeon performs acupotomy on the patient's suprapatellar sac, suprapatellar fat pad, inner knee eye, outer knee eye, and other parts, through cutting, separating, stripping, adjusting, and releasing tendon and ligament and other corresponding soft tissues, to relieve knee joint pain and improve joint function. $\mathrm{Li} \mathrm{Jia}$ et al.[33] compared the mRNA and protein expressions of TGF- $\beta 1$, Smad4, and matrix metalloproteinase-9 (MMP-9) in the knee ligament tissues of mice in the acupotomology group and other groups, and confirmed that acupotomology could protect damaged ligament tissues by regulating the secretion of TGF- $\beta 1$, Smd4, and MMP-9. Thus improving the prognosis of KOA.

\subsection{Herbal Fumigation}

"The Yellow Emperor Beijing, " said, "its evil, stain shape that sweat, evil can be solved with sweat", this method is to use decoction to make Traditional Chinese Medicine liquid in gasification through the skin, orifice, meridian point , and other ways go deep you Rationale, thus dredging channels and collisions, reconcile Yin and Yang. Liu Jing et al.[34] combined the treatment of KOA with sodium hyaluronate injection and Traditional Chinese Medicine fumigation and found that the treatment efficiency reached $6.5 \%$. Therefore, it was concluded that the treatment of patients with knee osteoarthritis with Traditional Chinese Medicine fumigation could achieve good results and effectively relieve their joint pain.

\subsection{Auricular Point Bolus Method}

The method of ear-point pill pressing is to place the seeds and other pills in the ear-point and continue to stimulate the acupoints to prevent and treat diseases. Wang Jun et al.[35] added the ear acupoint pressing method on the basis of Traditional Chinese Medicine prescription and acupuncture treatment and found that CRP and ESR levels of patients could be reduced. Huang Yi et al.[36] applied ear point bean pressing combined with Traditional Chinese Medicine fumigating to treat early and middle stage knee osteoarthritis, and applied ear point bean pressing combined with Traditional Chinese Medicine fumigating to sympathetic, Shenmen, and subcortical points, and found that VAS pain score decreased and KSS knee score increased after treatment.
Auricular point pressing bean combined with Traditional Chinese Medicine fumigation therapy can significantly improve the patients' joint pain and joint activity.

\section{Summary and Outlook}

In conclusion, TCM external treatment has the advantages of clear curative effect, simple operation, and safety in the treatment of knee osteoarthritis. Meanwhile, TCM external treatment has a definite curative effect and obvious effect in alleviating the clinical symptoms of patients with knee osteoarthritis, delaying the progress of the disease, and improving the quality of life of patients.

\section{References}

[1] Liu Cunbin, Li Tao, Geng Kai, Lin Xiuhua, Wu Liangliang, Luo Shiyu, Yang Yonghui. Progress in the treatment of knee osteoarthritis[J]. Journal of Anhui University of Traditional Chinese Medicine, 2021, 41(01): 50-53.

[2] Hao Xin, Yu Ying, Qiao Hujun. Knee arthritis etiology diagnosis and exercise therapy[J]. Journal of Contemporary Sports Science and Technology, 2021, (9): 23-25.

[3] Zhou Shuaiqi, Liang Long, Yu Jie, Feng Minshan, Wei $\mathrm{Xu}$, Chen Ming, Zhan Jiawen, He He, Yin Xunlu, Zhu Liguo. Latest knee osteoarthritis evidence-based international guidelines for the survey[J]. Journal of Hainan Medical College, 2020, 26(5): 388-391.

[4] Tian Huiping, Liu Ying, Wang Haiyang, et al. Non-surgical treatment of proliferative knee arthritis Rheumatoid Arthritis, 2018, 7(01): 67-71.

[5] Zhang Wenqiang, Ding Qian, Zhang Na. Combined treatment of Traditional Chinese Medicine in the treatment of knee osteoarthritis application effect analysis[J]. Journal of Liaoning Traditional Chinese Medicine Magazine, 2016(8): 1643-1645.

[6] Chen W H. Guidance on TCM diagnosis and treatment of knee osteoarthritis (2020 edition)[J]. Chinese Traditional Bonesetting, 2020, 32(10): 1-14.

[7] Tu Jianfeng, Wang Liqiong, Shi Guangxi, Yang Jingwen, Li Jinling, Li Yongting, Zhao Jingjie, Hou Haikun, Du Yi, Liu Cunzhi. Effect of acupuncture on knee joint injury and osteoarthritis score in patients with knee osteoarthritis $[\mathrm{J}]$. Chinese Acupuncture and Moxibustion, 2021, 41(01): 27-30.

[8] Yin Yueshan, Ruan Anmin, Zhao Wanming, Ma Yufeng, Zhao Jiping. Clinical observation of electroacupuncture in the treatment of knee osteoarthritis[J]. Chinese Journal of Orthopedics and Trauma, 2019, 27(12): $48-51+55$.

[9] Sangdee C, Teekachunhatean S, Sananpanich K, et al. Electroacupuncture versus diclofenac in the symptomatic treatment of osteoarthritis of the knee: A randomized controlled trial[J]. BMC Complement Altern Med, 2002, 2: 3.

[10] Ahsin S, Saleem S, Bhatti AM, et al. Clinical and endocrinological changes after electroacupuncture treatment in patients with osteoarthritis of the Knee[J]. Journal of Pain, 2009, 147(3): 60-66.

[11] Gang Jiahong, Mi Yiqun, Wang Huamin. Clinical efficacy of electroacupuncture and meloxicam in 
treatment of early and middle stage knee osteoarthritis: a randomized controlled study $[\mathrm{J}]$. Chinese Acupuncture, 2016, 36(5): 467-470.

[12] Pan Rong, Chen Yiming. Clinical efficacy of electroacupuncture combined with functional exercise and external application of Traditional Chinese Medicine in the treatment of knee osteoarthritis[J]. Health Medicine Research \& Practice, 2021, 18(04): 86-90.

[13] Fu Erun, Chen Junwei, Li Bin, Yuan Fang, Sun Jingqing. Treatment of knee osteoarthritis with fire needle and its effect on serum inflammatory cytokines[J]. Chinese Acupuncture, 2021, 41(05): 493-497.

[14] Xie Chuan, XIE Weina. Clinical effect of Shaolin acupuncture on knee osteoarthritis[J]. Clinical research of Traditional Chinese Medicine, 2020, 12(23): 90-93.

[15] Song Weiping. Yang deficiency type hemorheological nature Mlli fire needle treatment of knee osteoarthritis clinical observation[J]. Journal of Traditional Chinese Medicine in Yunnan, 2020, 9(6): 64-66.

[16] Chen Yu, Wang Ruiqing, Liu Jingxuan, Zhang Zidi, Jia Yuan, Lv Jiuheng, Shi Jing, Xu Jing, Jia Chunsheng. Effects of moxibustion on inflammatory factors and oxidative stress factors in patients with knee osteoarthritis: a randomized controlled study[J]. Chinese Acupuncture and Moxibustion, 2020, 40(09): 913-917.

[17] Yang Liu, Chen Xinhua. Moxibustion mechanism research progress for the treatment of knee osteoarthritis[J]. Chinese Folk Therapy, 2021, 29(4): 115-118.

[18] Zhang S N, Ouyang L Z, Wang X Z, et al. Effects of Acupuncture combined with thunder and fire moxibustion on map-3, timp-1, and TGF- $\beta 1$ in rats with knee osteoarthritis[J].Journal of Acupuncture and Tuina Science, 2017, 15(05): 322-327.

[19] Zhang Hanyi, Xu Daoming, Xue Liang, et al. Clinical study of thunder fire moxibustion combined with celecoxib in the treatment of knee osteoarthritis[J]. Journal of Nanjing University of Traditional Chinese Medicine, 2017, 33(06): 574-578.

[20] Zhao Min, Geng Jie, Feng Wei. Cassia twig peony rhizoma anemarrhenae tonga subtraction joint thunder fire moxibustion treatment of knee osteoarthritis clinical research[J]. Journal of New Traditional Chinese Medicine, 2021, 2(13): 27-31.

[21] Tian Aiping, Xu Lei, Song Qiumei, Lu Huichao, Chi Mingchun. Effects of thunder and fire moxibustion combined with Duhuazi Decoction on knee function, pain and serum inflammatory factors in patients with knee osteoarthritis[J]. Journal of Guangzhou University of Chinese Medicine, 2021, 38: 961-967.

[22] Cui Fan, Sun Yan, Li Yutang. Clinical observation on treatment of knee osteoarthritis with thunder fire moxibustion combined with sodium hyaluronate injection[J]. Chinese Folk Therapy, 2019, 27(23): 23-25.

[23] Hu Jun, Liu Lanying, Wang Hesheng, Zhang Cong, Du Lia, Zhang Xuemei, Yu Xiangjiang. Evaluation of the synergistic effect of thunder fire moxibustion in treatment of knee osteoarthritis[J]. Clinical Journal of Traditional Chinese Medicine, 2019, 31(08): 1557-1560.

[24] Chen Rixin, Xie Dingyi. Theoretical system construction and clinical application of heat sensitive moxibustion[J]. World Chinese Medicine, 2019, 14(8): 1915-1921.
[25] Wang Wenxue. Thermal moxibustion treatment wind cold dampness type certification of knee osteoarthritis clinical efficacy[J]. Journal of Clinical Medicine Literature Electron, 2018, 5(60): 58.

[26] Fang Xianfeng, Zhu Mengji, Liang Jinzhen. Thermal moxibustion with oral medicine clinical observation on treatment of knee osteoarthritis[J]. Journal of Practical Combine Traditional Chinese and Western Medicine Clinical, 2018, 18(9): 6-8.

[27] Chen Yong, Chen Bo, Chen Zelin, et al. Clinical and biological mechanism of cupping therapy[J]. World Traditional Chinese Medicine, 2020, 15(11): 1643-1650.

[28] Qiu Jianqing, Liu Shuru, Lin Qianlin, Li Mingjing, Zhuang Jingxiang, $\mathrm{Wu}$ Guangwen. Acupuncture combined with cupping therapy for knee osteoarthritis with qi stagnation and blood stasis: a randomized controlled study[J]. Chinese Acupuncture and Moxibustion, 2019, 39(05): 462-466.

[29] Jiang Liming, Zhang Zengqiao, Feng Wei. Clinical effect of releasing the subpatellar fat pad in treatment of knee osteoarthritis[J]. Chinese Journal of Gerontology, 2020, 40(02): 342-345.

[30] Qu Liuxin, Wang Luye, Xing Liyang, et al. Clinical study of chiropractic therapy in the treatment of knee osteoarthritis[J]. Journal of Nanjing University of Traditional Chinese Medicine, 2019, 35(02): 152-155.

[31] $\mathrm{Hu} \mathrm{Qu}$, Zeng Zhen, Hu Guangyun, et al. Etiological diagnosis and exercise therapy of knee arthritis[J]. Chinese Journal of Acupuncture and Moxibustion, 2019, 39(08): 804-808.

[32] Wang Zheng, Liu Anping, Liang Wenwu, et al. Clinical study of Traditional Chinese Medicine in the treatment of knee osteoarthritis[J]. Journal of Anhui University of Traditional Chinese Medicine, 2019, 38(04): 28-32.

[33] Li Jiaru, Cao Yunming, Yang Yupeng, SI Jiahong, Zhao Jiyu, Ren Huashan, Jin Xiaofei, Yan Ping. Effects of acupotomy on TGF- $\beta 1 /$ Smads signaling pathway in knee osteoarthritis rabbits $[\mathrm{J}]$. Acupuncture Clinical Journal, 2021, 5(12): 70-75.

[34] Eppinger S. The Traditional Chinese Medicine fumigation treatment of knee osteoarthritis clinical observation $[\mathrm{J}]$. Journal of Foot and Health Care, 2018, 27(24): 123-124.

[35] Wang Jun, Wang Shuai, Wang Yanjun. Clinical observation of auricular acupoint pressing bean combined with Chinese herbal prescription and acupuncture in treatment of knee osteoarthritis[J]. Guangming Traditional Chinese Medicine, 2019, 34(06): 917-919.

[36] Huang Yi, Yuan Jiajun, Tong Peijian. Treatment of knee osteoarthritis in patients with early and middle stage[J]. Chin J Orthopaedics, 2018, 26(03): 55-56+60. 